\title{
Design of a broadband Ka-band MMIC LNA using deep negative feedback loop
}

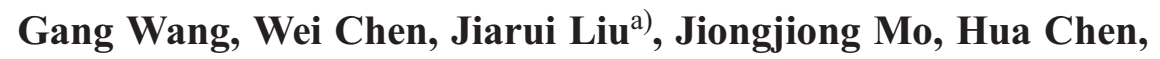 \\ Zhiyu Wang, and Faxin Yu \\ School of Aeronautics and Astronautics, Zhejiang University, \\ 38 Zheda Road, Hangzhou, China \\ a) jrliu@zju.edu.cn
}

Abstract: In this paper, we present a broadband Ka-band LNA using 0.15- $\mu \mathrm{m}$ GaAs pseudomorphic high electron mobility transistor (pHEMT) process. By using bandwidth enhancement techniques and deep negative feedback technology, the LNA achieves relatively broadband performances. The LNA attains $20 \mathrm{~dB}$ small signal gain from 25 to $40 \mathrm{GHz}$ and shows a measured noise figure of $2.8 \mathrm{~dB}$ from 25 to $40 \mathrm{GHz}$ with $230-\mathrm{mW}$ dc power consumption. The input and output return loss of the LNA is less than $8 \mathrm{~dB}$, which is competitive compared with other published Ka-band LNAs. The size of the chip is $2.5 \mathrm{~mm} \times 1.2 \mathrm{~mm}$.

Keywords: Ka-band, low noise amplifier (LNA), MMIC, broadband Classification: Microwave and millimeter-wave devices, circuits, and modules

\section{References}

[1] European Southern Observatory website: http://www.eso.org/public/teles-instr/ alma/.

[2] G. Gonzalez: Microwave Transistor Amplifier Analysis and Design (Pearson Prentice, 1996) 2nd ed.

[3] D. Shaeffer, et al.: "A 1.5-V, 1.5-GHz CMOS low noise amplifier," IEEE J. Solid-State Circuits 32 (1997) 745 (DOI: 10.1109/4.568846).

[4] H. P. Moyer, et al.: "Q-band GaN MMIC LNA using a $0.15 \mu \mathrm{m}$ T-Gate process," Proc. IEEE CSIC Symposium Digest (2008) 99 (DOI: 10.1109/ CSICS.2008.26).

[5] R. Malmqvist, et al:: "Design, packaging and reliability aspects of RF-MEMS circuits fabricated using a GaAs MMIC foundry process technology," 2010 European Microwave Integrated Circuits Conference (2010) (DOI: 10.23919/ EUMC.2010.5616523).

[6] Y.-T. Chou, et al.: "A Q-band LNA with 55.7\% bandwidth for radio astronomy applications in $0.15-\mu \mathrm{m}$ GaAs pHEMT process," IEEE International Symposium on Radio-Frequency Integration Technology (2016) 1 (DOI: 10. 1109/RFIT.2016.7578197).

[7] R. Limacher, et al:: "Broadband low-noise amplifiers for K- and Q-band using $0.2 \mu \mathrm{m}$ InP HEMT MMIC technology," Proc. IEEE CSIC Symposium Digest (2004) 305 (DOI: 10.1109/CSICS.2004.1392575).

[8] S.-H. Weng, et al:: "Q-band low noise amplifiers using 0.15- $\mu \mathrm{m}$ MHEMT process for broadband communication and radio astronomy applications," 
IEEE MTT-S International Microwave Symposium Digest (2008) 455 (DOI: 10.1109/MWSYM.2008.4633201).

[9] S.-H. Weng, et al.: "Cryogenic evaluation of a $30-50 \mathrm{GHz} 0.15-\mathrm{m}$ MHEMT low noise amplifier for radio astronomy applications," IEEE 41st European Microwave Conference (2011) 934 (DOI: 10.1109/RFIT.2012.6401652).

[10] H.-C. Yeh, et al:: "Analysis and design of millimeter-wave low-voltage CMOS cascade LNA with magnetic coupled technique," IEEE Trans. Microw. Theory Techn. 60 (2012) 4066 (DOI: 10.1109/TMTT.2012.2224365).

[11] P.-H. Ho, et al.: "An ultra low-power Q-band LNA with 50\% bandwidth in WIN GaAs $0.1-\mu \mathrm{m}$ pHEMT process," Asia-Pacific Microwave Conference Proceedings (APMC) (2013) 713 (DOI: 10.1109/APMC.2013.6694906).

\section{Introduction}

The Atacama large millimeter/sub-millimeter array (ALMA) is a state-of-art ground based astronomical telescope projected to explore the outer space in the millimeter and sub-millimeter regime. It enables scientists to conduct further research into the building blocks of stars, planetary systems, galaxies and life itself by sensing detail images of planets and galaxies in the observable universe [1].

To improve the image resolution with the weak receiving power, a highly sensitive receiver system is required. The signal-to-noise ratio of the overall receiver is dominated by the noise and gain of the first-stage low noise amplifier (LNA). Most of the LNAs with high gain and low noise performance are designed in GaAs- or InP-based HEMT processes. Although the InP-based HEMT devices demonstrate better millimeter wave (MMW) characteristics than GaAs HEMT devices, GaAs HEMT is a mature microwave integrated circuit process that strikes a balance between circuit performance and fabrication cost [2].

In this paper, we present a Ka-band LNA for radio astronomy applications using $0.15-\mu \mathrm{m}$ GaAs pHEMT process. The design targets of the LNA are 20-dB small signal gain and 3-dB noise figure to make sure that the total noise performance of the receiver system is guaranteed. Compared with the reported LNAs, this work shows high gain and low noise performances in Ka-band with acceptable dc power consumption.

\section{Design principle and approach}

The design of broadband LNA comprises two basic parts, the basic targets of the LNA and the bandwidth enhancement methods. The LNA adopts deep negative feedback loop to help enhancing the stability and broadening the bandwidth.

\subsection{Basic design concern}

The overall schematic of the Ka-band LNA is shown in Fig. 1. The LNA is based on four-stage common source structure with the matching networks realized by microstrip lines and reference ground using backside metal.

Stability is an important figure of merit for any amplifier design. In order to achieve unconditional stability, it is extremely important that the matching is done properly, otherwise large standing waves will appear. The large standing waves will 
not only destroy the amplifier performance but also result in amplifier instability which makes the amplifier unusable at all [3]. Other than doing the input and output matching networks in a multi-stages LNA, it is also important that the inter-stage matching networks are properly designed as well.

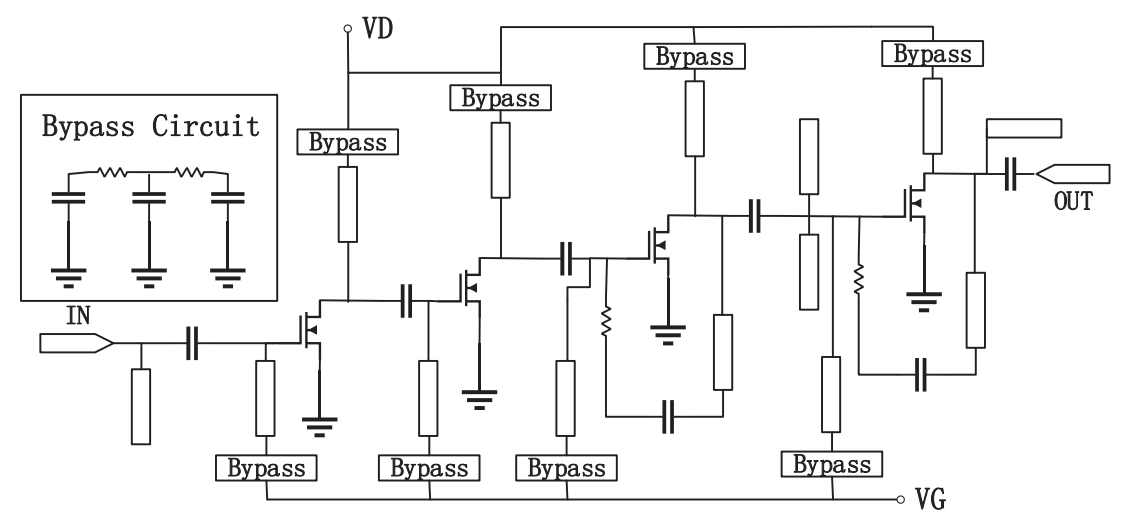

Fig. 1. Circuit configuration of the LNA.

A two-finger transistor with total gate width of $100 \mu \mathrm{m}$ is applied to four stages for sufficient gain and low noise figure. The major concern of the input matching is to minimize the noise figure while sustaining enough power gain. It is well-known that source degeneration technique can meet the goal by making the minimum noise figure point $\left(\boldsymbol{\Gamma}_{\mathrm{opt}}\right)$ and input conjugate point $\left(\boldsymbol{\Gamma}_{\mathrm{MS}}\right)$ point close to each other on Smith chart [4]. In this work, two $100-\mu \mathrm{m}$ microstrip lines are connected to the source of the first-stage transistor. In addition, with the source degeneration and the loss of the matching networks, the circuit can be stabilized. A good design will have an amplifier with minimum noise figure at first stage and low-pass network at the output in order to reduce the spurious and high harmonic content. Band-pass networks at the input and inter-stages will provide good broadband matching and gain forming to increase the stability.

\subsection{Bandwidth enhancement techniques}

To broaden the bandwidth of the LNA, two techniques are used. One is the $\pi$-type matching network as shown in Fig. 2. Since an open stub is equivalent to a shunt capacitor and a short stub is equivalent to a shunt inductor, the $\pi$-type matching network can be thought as a LC tank [5]. By lowering the Q factor of a LC tank, bandwidth can be extended. The other technique is the deep negative feedback loop. The resistor, capacitor and microstrip between drain and gate of the transistor form a feedback network. The aim of the network is to mismatch inter-stage

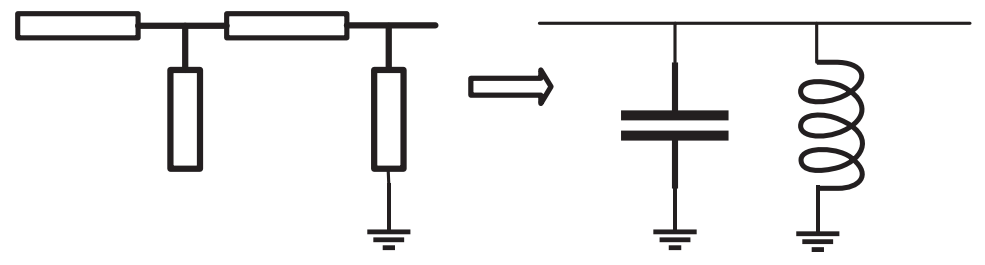

Fig. 2. $\pi$-type matching network 
matching networks to compensate for the changes with frequency of gain. In other words, the matching networks are designed to match at different frequency bands at different stages.

\section{Implementation and measurements}

The chip is fabricated with $0.15 \mu \mathrm{m}$ GaAs pHEMT process. Fig. 3 shows the photograph of the Ka-band LNA. The chip size is $2.5 \mathrm{~mm} \times 1.2 \mathrm{~mm}$. Dc bias is fed through bond pad, and the noise figure is measured by spectrum analyzer (R\&S FSU43). The S-parameters are tested by vector network analyzer (Agilent PNA 5224A) using GSG co-planar microwave probes on probe station.

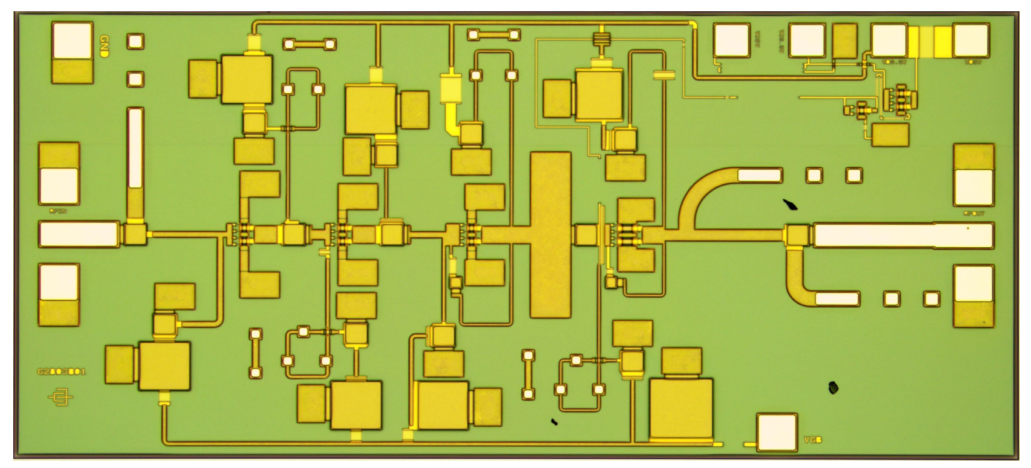

Fig. 3. Photograph of the fabricated LNA.

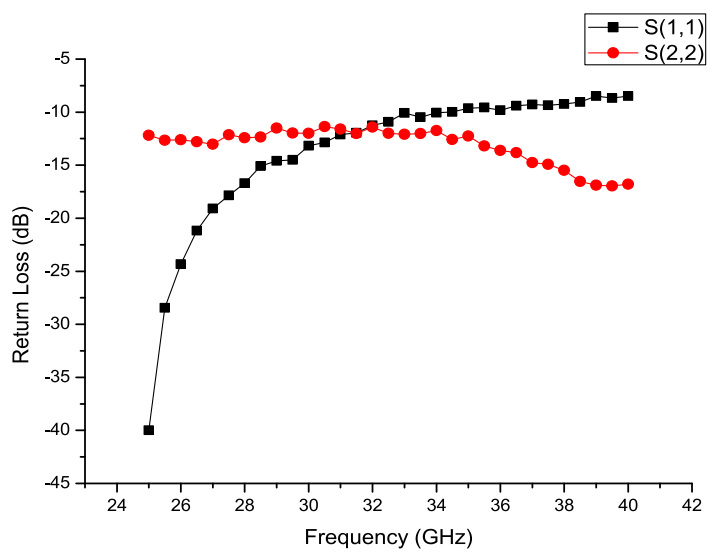

Fig. 4. Input and output return loss of the Ka-band LNA

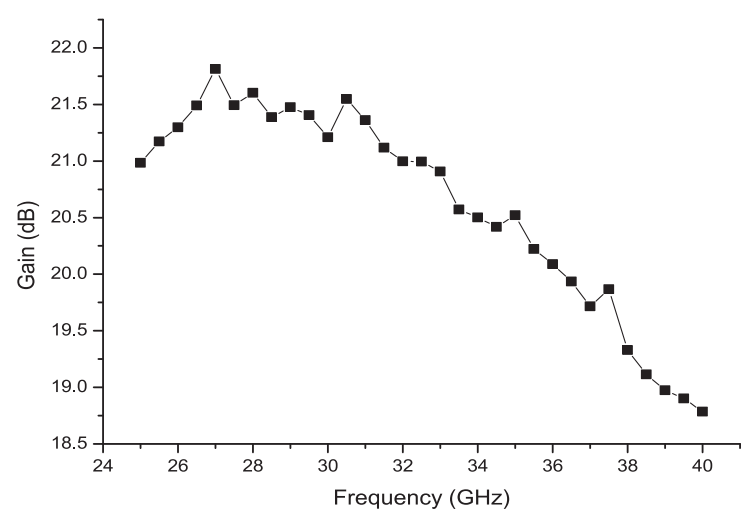

Fig. 5. Gain of the Ka-band LNA 


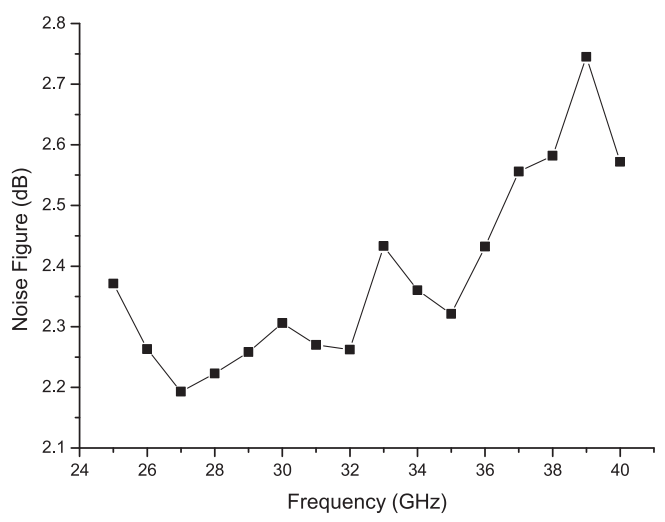

Fig. 6. Noise figure of the Ka-band LNA

As shown in Fig. 4, the fabricated Ka-band LNA exhibits less than $-8 \mathrm{~dB}$ input and output return loss at frequency ranging from 25 to $40 \mathrm{GHz}$. Fig. 5 demonstrates the average gain of $20 \mathrm{~dB}$ at this frequency range. Fig. 6 shows the noise figure of the LNA in the whole band, which is less than $2.8 \mathrm{~dB}$. The bias voltage of $\mathrm{Vd}$ is $5 \mathrm{~V}$ and the working current is $46 \mathrm{~mA}$. Table I summarizes the performance comparison of this work with other published LNAs working at close frequency ranges. The characteristics of the LNA includ return loss, gain, noise figure and total chip area consume.

Table I. Performance comparison of relevant LNAs

\begin{tabular}{c|c|c|c|c|c|c}
\hline Ref & $\begin{array}{c}\text { Device } \\
\text { Tech }\end{array}$ & $\begin{array}{c}\text { Freq } \\
(\mathrm{GHz})\end{array}$ & $\begin{array}{c}\text { Gain } \\
(\mathrm{dB})\end{array}$ & $\begin{array}{c}\text { Noise } \\
\text { Figure } \\
(\mathrm{dB})\end{array}$ & $\begin{array}{c}\text { Return } \\
\text { Loss } \\
(\mathrm{dB})\end{array}$ & $\begin{array}{c}\text { Size } \\
\left(\mathrm{mm}^{2}\right)\end{array}$ \\
\hline$[6]$ & $\begin{array}{c}0.15-\mu \mathrm{m} \\
\text { GaAs pHEMT }\end{array}$ & $28.5 \sim 50.5$ & 23 & 3.8 & 5 & $2.0 \times 1.5$ \\
\hline$[7]$ & $\begin{array}{c}0.2-\mu \mathrm{m} \\
\text { InP HEMT }\end{array}$ & $23 \sim 49$ & 11 & 2.5 & 5.5 & $2.3 \times 1.5$ \\
\hline$[8]$ & $\begin{array}{c}0.15-\mu \mathrm{m} \\
\text { GaAs } \\
\mathrm{mHEMT}\end{array}$ & $32 \sim 50$ & 29 & 3.1 & 5 & $2.0 \times 1.0$ \\
\hline$[9]$ & $\begin{array}{c}0.15-\mu \mathrm{m} \\
\text { GaAs } \\
\mathrm{mHEMT}\end{array}$ & $30 \sim 50$ & 20 & 3.7 & 4 & $2.0 \times 1.0$ \\
\hline$[10]$ & $\begin{array}{c}90-\mathrm{nm} \\
\text { CMOS }\end{array}$ & $30 \sim 42$ & 18 & 5 & 7 & $0.6 \times 0.48$ \\
\hline$[11]$ & $\begin{array}{c}0.1-\mu \mathrm{m} \\
\text { GaAs pHEMT }\end{array}$ & $27 \sim 45$ & 25 & 3.1 & 5 & $2.0 \times 1.0$ \\
\hline $\begin{array}{c}\text { This } \\
\text { work }\end{array}$ & $\begin{array}{c}0.15-\mu \mathrm{m} \\
\text { GaAs pHEMT }\end{array}$ & $25 \sim 40$ & 20 & 2.8 & $>8$ & $2.5 \times 1.2$ \\
\hline
\end{tabular}

\section{Conclusion}

In this letter, a high-performance broadband Ka-band LNA is reported. The MMIC LNA is fabricated with $0.15-\mu \mathrm{m}$ GaAs pHEMT process. A deep negative feedback topology is employed in the last two stages to extend the bandwidth and to achieve 
stability. The measurement data reveals that the LNA has $20 \mathrm{~dB}$ gain, and more than $8 \mathrm{~dB}$ input and output return loss. Noise figure in the whole working frequency is less than $2.8 \mathrm{~dB}$. The measured results show low noise figure and high gain of the presented monolithic LNA.

\section{Acknowledgments}

This work was supported by the National Science Foundation of China under Grant 61401395 and 61604128, the Scientific Research Fund of Zhejiang Provincial Education Department under Grant Y201533913, and the Fundamental Research Funds for the Central Universities under Grant 2016QNA4025. 